\title{
Culture and Diet Among Chinese American Children Aged 9-13 Years: A Qualitative Study
}

\author{
Cassandra S. Diep, $\mathrm{PhD}^{1,2}$; Randall Leung, $\mathrm{BS}^{3}$; Debbe I. Thompson, $\mathrm{PhD}, \mathrm{RD}^{1}$; \\ Beverly J. Gor, EdD, RD, LD $^{4}$; Tom Baranowski, $\mathrm{PhD}^{1}$
}

\begin{abstract}
Objective: To examine Chinese American children's behaviors, food preferences, and cultural influences on their diet.

Design: Qualitative individual interviews using constructs from the proposed model of dietary acculturation. Setting: Community centers and Chinese schools in Houston, TX.

Participants: Twenty-five Chinese American children aged 9-13 years.

Phenomenon of Interest: Diet, favorite restaurants, and parents' cooking and grocery shopping habits. Analysis: Content analysis and thematic data analysis to identify code categories and themes. Coders also identified patterns based on demographic and acculturation factors.

Results: Overall, participants described their diets and associated behaviors as Asian and non-Asian. Key themes included preference for Asian and non-Asian foods; consumption of non-Asian foods for breakfast and lunch, but Asian foods for dinner; infrequent dining at restaurants; grocery shopping at Asian and nonAsian stores; and familial influences on diet. Acculturated children and children of higher socioeconomic status appeared to prefer and consume a more Westernized/non-Asian diet.

Conclusions and Implications: Results illustrate that Chinese American children in this study practiced both Asian and non-Asian dietary behaviors. Findings corroborated existing acculturation research with parents and caregivers; supported constructs in the model of dietary acculturation; and provide guidance for research and programs related to dietary behaviors, determinants, and culture among this population. Key Words: diet, Chinese, children, culture, food preferences, interviews, acculturation (J Nutr Educ Behav. 2017;49:275-284.)
\end{abstract}

Accepted November 6, 2016. Published online December 24, 2016.

\section{INTRODUCTION}

Chronic diseases such as heart disease and cancer are major public health concerns and represented 7 of the 10 leading causes of death in the US in $2010 .{ }^{1}$ Diet has an important role in many of these diseases. ${ }^{2,3}$ Because dietary behaviors formed during childhood may carry into adulthood ${ }^{4}$ and influence risk for chronic diseases later in life, ${ }^{5}$ nutrition research and interventions among children are needed.

One crucial population for such research is that of Asian descent, the fastest-growing immigrant group in

\footnotetext{
${ }^{1}$ US Department of Agriculture/Agricultural Research Service Children's Nutrition Research Center, Department of Pediatrics, Baylor College of Medicine, Houston, TX

${ }^{2}$ Department of Health Disparities Research, University of Texas MD Anderson Cancer Center, Houston, TX

${ }^{3}$ Department of Health Sciences, Boston University, Boston, MA

${ }^{4}$ Office of Planning, Evaluation and Research for Effectiveness, Houston Health Department, Houston, TX

Conflict of Interest Disclosures: The authors' conflict of interest disclosures can be found online with this article on www.jneb.org.

Address for correspondence: Cassandra S. Diep, PhD, Department of Health Disparities Research, Unit 1440, UT MD Anderson Cancer Center, PO Box 301402, Houston, TX 77230-1402; Phone: (713) 794-5007; Fax: (713) 792-1152; E-mail: CSYeung@, mdanderson.org

(C2016 Society for Nutrition Education and Behavior. Published by Elsevier, Inc. All rights reserved.

http://dx.doi.org/10.1016/j.jneb.2016.11.002
}

the US. ${ }^{6}$ Although rates of chronic diseases are relatively low among recent Asian immigrants, ${ }^{7}$ there is increasing disease risk among Asian American populations. For example, Asian American adolescents born in the US had greater obesity risk than did first-generation residents, with obesity rates similar to nonimmigrant youth. ${ }^{8}$ Furthermore, the risk markers of type 2 diabetes and metabolic syndrome are evident at lower body mass index levels among Asian populations compared with nonHispanic white peers. ${ }^{9,10}$ Traditional Asian diets, which are rich in fruit and vegetables, may protect against many chronic diseases, ${ }^{11}$ but qualitative research with Asian American primary caregivers showed that their children preferred and consumed more Western foods (eg, sweetened beverages, pizza) than did the primary caregivers. ${ }^{12-14}$

Of all Asian subgroups, the Chinese American subgroup is the largest and one of the longest-standing in the US. In 2013, Chinese American people accounted for $23.9 \%$ of the 
Asian-American population, with a population over 4.5 million. ${ }^{15}$ Previous research revealed that Chinese American individuals demonstrated changes in dietary patterns, $^{12,14,16,17}$ increased cardiovascular disease risks, ${ }^{18}$ and increased obesity risk ${ }^{19}$ across subsequent generations and over time. These patterns may be partially explained by dietary acculturation, or the process by which members of 1 group adopt the eating patterns of a new environment. ${ }^{17,20}$

With increasing numbers of Chinese American people living in the US, it is important to understand their dietary behaviors and determinants, including those related to dietary acculturation and culture, at an early age. Prior research in this area sampled primary caregivers ${ }^{12-14}$; research directly involving Chinese American children is lacking and needed. Children's accounts may complement caregivers' accounts by providing insight into children's food preferences, perspectives about what they eat and who influences that, and understandings about food. ${ }^{21-23}$ In addition, parental observations about their children may differ from children's perceptions, so that proxy information may be inadequate or inaccurate. ${ }^{24}$ Children may hide behaviors from their parents or have influences outside their parents and home environment. ${ }^{25,26}$

The purpose of this study was to better understand Chinese American children's behaviors, food preferences, and cultural influences on diet through qualitative interviews. The proposed model of dietary acculturation (MDA), which was conceptualized and illustrated by Satia-Abouta et al, 17,20 provided a comprehensive framework for this study. Constructs of the MDA include socioeconomic and demographic factors (eg, sex, age, household income), cultural factors (eg, cultural beliefs, living in an ethnic neighborhood), psychosocial factors (eg, dietrelated knowledge), taste preferences, and environmental factors (eg, shopping, restaurants), with interactions and changes in these constructs leading to different patterns of dietary intake.

\section{METHODS}

\section{Participants and Recruitment}

A convenience sample of Chinese American children aged 9-13 years was recruited through Houston-area com- munity/cultural settings (ie, Chinese language schools, community centers) and the Children's Nutrition Research Center's volunteer database. For in-person recruitment, study staff distributed information packets to parents and/or children to review at home. For recruitment through the volunteer database, parents were contacted and then mailed an information packet. Information packets included a flyer, information sheet, demographics questionnaire, study eligibility questionnaire, and consent form. If the parent and child agreed to be in the study, they provided written informed consent and assent, completed the questionnaires, and returned the completed forms to study staff by mail or for pickup at the community/ cultural setting.

Eligibility criteria included (1) selfidentification as Chinese or Taiwanese (either full or part) and (2) age 913 years. This age range was chosen to understand better the transition from late elementary to middle school, which represents a developmental period critical for nutrition education and promotion, $^{27,28}$ and which may be an ideal opportunity for influencing dietary change. ${ }^{28}$ Children were excluded if they could not speak, read, and write English, or if they had a limitation that would inhibit their ability to complete an interview or influence their diet or physical activity. Of 27 children who agreed to participate, 2 did not meet eligibility criteria, which resulted in 25 children in the study. Enrollment and interviews were continued until data saturation, or the point at which no new information emerged, as deemed by the interviewer. Data collection ceased after several interviews with redundant information.

\section{Procedures}

The first author, who was trained in qualitative data collection methods, interviewed each child privately over the phone $(n=10)$ or in person $(n=15)$. Before each interview, the interviewer explained the purpose of the interview (ie, to learn more about the child's cultural identity and diet). Each interview followed a semistructured interview guide with open-ended questions; probes were also used to elicit clarification or additional responses. All inter- view questions were developed by the first author based on constructs from the $\mathrm{MDA}^{17,20}$ and were approved by the coauthors. Table 1 includes interview questions and associated MDA constructs. All interviews were conducted between October 2014 and July 2015, and lasted an average of 14 minutes.

Participants also completed a revised Suinn-Lew Asian Self-Identity Acculturation scale (SL-ASIA). ${ }^{29}$ The SL-ASIA, which was originally developed for adults, was modified for children by the current authors before this study. Revisions were based on cognitive interviews with a different sample of Chinese American children aged 10-13 years $(n=9)$, each of whom read through directions, items, and responses and provided feedback on comprehension. Revisions included changing words that children did not understand (eg, Oriental), making response categories collectively exhaustive, and revising phrasing that confused children. For example, response options for How would you rate yourself? were changed from very Asian, mostly Asian, bicultural, mostly Westernized, and very Westernized to very Asian, mostly Asian, both Asian and Americanized (bicultural), mostly Americanized, and very Americanized.

Each child received a $\$ 40$ gift card for completing the interview and revised SL-ASIA. All study procedures were approved by the Institutional Review Board of Baylor College of Medicine (Protocol H-32020).

\section{Data Analysis}

Interviews were audio-recorded and transcribed verbatim. Transcriptions were double-checked against the original recordings, imported into NVivo qualitative data analysis software (Version 10.0, 2012, QSR International Pty Ltd, Doncaster, Victoria, Australia), and analyzed using a hybrid approach ${ }^{30}$ of content analysis ${ }^{31}$ directed by the MDA and thematic data analysis. ${ }^{32}$ First, 5 code categories, which reflected the MDA and questions in the interview guide, were selected: favorite foods and drinks, parents' cooking (later expanded to typical foods), restaurants, grocery stores, and influences on diet. Thematic analysis was then conducted to identify themes that emerged within these 


\section{Question}

Questionnaires

Is your child a boy or a girl?

Child's date of birth

What is the highest education level finished among all people living in your child's home?

What was the approximate total income, before taxes, of the child's household in 2013?

Where was your child born?

How would you rate yourself?

Interview

1. What are your favorite foods or drinks?

1a. Do you think the foods you eat and the foods you like are more Asian, nonAsian, both, or neither?

1b. What do you usually eat each day?

1c. Do you think what you eat is the same or different from what your friends eat?

2. When you eat at home, what do your parents usually cook?

3. What are your favorite restaurants?

3a. What foods or drinks do you eat there?

4. Where do your parents usually shop for food?

4a. What foods do they buy there?

4b. What foods or drinks do you almost always have at home?

5. What or who do you think most affects what you eat?

6. Before we end, is there anything else you want to say about being Asian or American, about the foods you eat, or anything else?

\section{MDA Construct}

Socioeconomic and demographic factors

Socioeconomic and demographic factors

Socioeconomic and demographic factors

Socioeconomic and demographic factors

Socioeconomic and demographic factors; cultural factors

Socioeconomic and demographic factors; cultural factors

Psychosocial factors and taste preferences; dietary intake

Psychosocial factors and taste preferences; dietary intake

Dietary intake

Psychosocial factors and taste preferences; dietary intake

Environmental factors; dietary intake

Environmental factors

Environmental factors; dietary intake

Environmental factors

Environmental factors

Environmental factors

$\mathrm{N} / \mathrm{A}$

$\mathrm{N} / \mathrm{A}$

N/A indicates not available.

categories. For example, responses for What are your favorite foods or drinks? were coded under favorite foods and drinks as Asian, non-Asian, none (ie, not having a favorite food or drink), and shared by both cultures/ambiguous.

Two coders (the first and second authors) were trained in NVivo and qualitative data analysis by a colleague, who was experienced in qualitative research methods. Before coding began, both coders discussed the 5 code categories and potential themes. Both coders independently coded the data for 21 transcripts $(84 \%)$ to minimize potential biases; codings were compared and any differences were reviewed and resolved. The first author coded the remaining 4 transcripts. A code manual was emergent and created during coding. The first author also completed additional readings of all interview transcripts, comparing and contrasting them with the code manual to confirm or modify themes. A final read found no additional themes.
Initial coding of meaning segments included demographic and acculturation characteristics. To identify themes or patterns based on these factors, a matrix coding query in NVivo was executed. Demographics included child's sex (boy or girl), age $(9,10,11,12$, or 13 years), and socioeconomic status (SES) (low or high) based on the parentcompleted demographics questionnaire included in the information packet. Low SES was classified as those from households with an annual income of $<\$ 40,000$ and/or no college education, similar to other low-income and education categories. ${ }^{33,34} \mathrm{~A}$ household income of $\$ 40,000$ approximately corresponded to the income eligibility for a 3-person family for free or reducedprice school meals in 2014-2015. ${ }^{35}$ Acculturation was measured using 2 separate items: (1) child's birthplace (Asian-born or American-born) from the demographics questionnaire and (2) child's self-identified cultural rating (How would you rate yourself?) from the revised SL-ASIA. The latter was coded as Asian-identified, bicultural, and American-identified.

\section{RESULTS}

A total of 25 children completed the study. All children were aged 9-13 years; nearly one third (32\%) were aged 10 years. Participants were about equal boys (52\%) and girls (48\%), and most were Chinese or Taiwanese alone $(88 \%)$, born in the US or Canada (64\%), self-identified as bicultural $(52 \%)$, and lived in a household with at least 1 parent completing postgraduate study (60\%). Three children were of multiple races/ethnicities, including Taiwanese/white and Chinese/Hispanic. Table 2 details demographic characteristics.

\section{Favorite Foods and Drinks}

When asked to describe their favorite foods, 15 children listed both Asian and non-Asian foods. Examples of Asian 
Table 2. Demographic Characteristics of Chinese American Children Completing Interviews $(n=25)$

\begin{tabular}{|c|c|c|c|c|c|c|}
\hline \multirow[b]{2}{*}{ Demographic Characteristics } & \multirow[b]{2}{*}{$\begin{array}{l}\text { Total } \\
\text { n (\%) }\end{array}$} & \multicolumn{2}{|c|}{ Birthplace } & \multicolumn{3}{|c|}{ Self-identified Cultural Rating } \\
\hline & & $\begin{array}{c}\text { US/Canada } \\
\text { n (\%) }\end{array}$ & $\begin{array}{c}\text { China/Taiwan } \\
\text { n (\%) }\end{array}$ & $\begin{array}{l}\text { Asian } \\
\text { n (\%) }\end{array}$ & $\begin{array}{c}\text { Bicultural } \\
\text { n (\%) }\end{array}$ & $\begin{array}{c}\text { American } \\
\text { n (\%) }\end{array}$ \\
\hline Total & $25(100)$ & $16(64)$ & $9(36)$ & $6(24)$ & $13(52)$ & $6(24)$ \\
\hline \multicolumn{7}{|l|}{ Age, y } \\
\hline 9 & $6(24)$ & $5(83)$ & $1(17)$ & $1(17)$ & $4(67)$ & $1(17)$ \\
\hline 10 & $8(32)$ & $4(50)$ & $4(50)$ & $3(38)$ & $3(38)$ & $2(25)$ \\
\hline 11 & $5(20)$ & $2(40)$ & $3(60)$ & $2(40)$ & $2(40)$ & $1(20)$ \\
\hline 12 & $3(12)$ & $2(67)$ & $1(33)$ & 0 & $2(67)$ & $1(33)$ \\
\hline 13 & $3(12)$ & $3(100)$ & 0 & 0 & $2(67)$ & $1(33)$ \\
\hline \multicolumn{7}{|l|}{ Sex } \\
\hline Boy & $13(52)$ & $10(77)$ & $3(23)$ & $3(23)$ & $6(46)$ & $4(31)$ \\
\hline Girl & $12(48)$ & $6(50)$ & $6(50)$ & $3(25)$ & $7(58)$ & $2(17)$ \\
\hline \multicolumn{7}{|l|}{ Race/ethnicity } \\
\hline Chinese or Taiwanese & $22(88)$ & $13(59)$ & $9(41)$ & $6(27)$ & $12(55)$ & $4(18)$ \\
\hline Multiple & $3(12)$ & $3(100)$ & 0 & 0 & $1(33)$ & $2(67)$ \\
\hline \multicolumn{7}{|l|}{ Highest household educational level } \\
\hline Some college or less & $6(24)$ & $2(33)$ & $4(67)$ & $1(17)$ & $5(83)$ & 0 \\
\hline College graduate & $4(16)$ & $2(50)$ & $2(50)$ & $2(50)$ & $1(25)$ & $1(25)$ \\
\hline Postgraduate study & $15(60)$ & $12(80)$ & $3(20)$ & $3(20)$ & $7(47)$ & $5(33)$ \\
\hline \multicolumn{7}{|l|}{ Household income } \\
\hline$<\$ 40,000$ & $7(28)$ & $2(29)$ & $5(71)$ & $3(43)$ & $4(57)$ & 0 \\
\hline$\$ 40,000$ to $\$ 69,999$ & $3(12)$ & $1(33)$ & $2(67)$ & 0 & $2(67)$ & $1(33)$ \\
\hline$\$ 70,000$ to $\$ 99,999$ & $6(24)$ & $6(100)$ & 0 & $2(33)$ & $2(33)$ & $2(33)$ \\
\hline$\geq \$ 100,000$ & $8(32)$ & $6(75)$ & $2(25)$ & $1(13)$ & $5(63)$ & $2(25)$ \\
\hline Did not report & $1(4)$ & $1(100)$ & 0 & 0 & 0 & $1(100)$ \\
\hline
\end{tabular}

foods included rice, noodles, dumplings, milk tea with tapioca, Asian soups (eg, phở, ramen), sushi, Asian vegetables (eg, bok choy, bai cai), and Asian dishes (eg, sweet and sour chicken, Kung Pao Chicken). Examples of non-Asian foods included pizza, chicken nuggets, soda, hamburger, fries, hot chips (eg, Flamin' Hot Cheetos), and spaghetti. Foods that were ambiguous or shared by both cultures included meat, water, eggs, bread, milk, juice, fruit (eg, apples), and vegetables (eg, okra). Seven children (some overlapping and some separate from the 15 listed) mentioned liking more Asian foods, whereas 8 other children liked more non-Asian foods. Two children made the distinction between eating mostly food from 1 culture and liking food from a different culture. As 1 child said, "My main diet consists of rice yet the food I like to eat or I like is pizza," whereas another noted, "Even though I prefer more Asian food, there are a lot times where I eat more ... Americanized food."

All children who identified as Asian according to the SL-ASIA, as well as most bicultural children (77\%; $\mathrm{n}=10$ ), listed at least 1 Asian food as a favorite food. In comparison, 33\% of children who self-identified as American $(\mathrm{n}=2)$ listed Asian foods as a favorite.

\section{Typical Foods}

Typical foods and meals were examined through discussion of home-cooked foods and foods eaten daily. A prevalent theme was the consumption of rice and/ or noodles. Fifteen children said they ate rice and/or noodles every day or almost every day. Only 33\% of Americanidentified children $(n=2)$ ate rice and/ or noodles almost every day, compared with $83 \%$ of Asian-identified and $62 \%$ of bicultural children ( $\mathrm{n}=5$ and 8 , respectively).

Other general themes included consumption of non-Asian foods for breakfast, lunch occurring mostly in a school setting, and consumption of Asian foods for dinner (Table 3). Multiple children listed negative perceptions about school food, including not liking the foods served and long lunch lines. Three health reasons were mentioned, such as having food allergies and not wanting to risk getting sick at school, or describing the school food as "not like as clean."

Based on demographic and acculturation factors, themes emerged for breakfast, lunch, and dinner foods. Many participants born in the US (69\%; $\mathrm{n}=11)$ or of higher SES (59\%; $\mathrm{n}=10)$ listed non-Asian foods as breakfast items, vs $22 \%$ of Asian-born participants $(n=2)$ or $29 \%$ of lower-SES participants $(\mathrm{n}=2)$, respectively. There also appeared to be differences by sex (69\% of boys, $\mathrm{n}=9$; $33 \%$ of girls, $\mathrm{n}=4$ ) and self-identified cultural rating $(83 \%$ of American-identified, $\mathrm{n}=5 ; 54 \%$ of bicultural, $\mathrm{n}=7$; and $17 \%$ of Asianidentified, $\mathrm{n}=1$ ) for consumption of non-Asian breakfast items. Regarding lunch, $86 \%$ of children aged 9 and 10 (ie, elementary school students; $\mathrm{n}=12$ ) ate school lunch, compared with $36 \%$ of children aged 11,12 , and 13 (ie, middle school students; $\mathrm{n}=4$ ). In a related manner, $47 \%$ of higher-SES 


\section{Theme}

Typical Foods

Rice and/or noodles $(n=15)$

Non-Asian foods for breakfast $(n=13)$

Asian foods for breakfast $(n=6)$

School foods for lunch $(n=16)$

Lunches brought from home $(n=10)$

Asian foods for dinner $(n=18)$

Non-Asian foods for dinner $(n=3)$

Non-Asian drinks and snacks $(n=9)$

Restaurants

Non-Asian fast food $(n=10)$

Ambiguous Asian restaurants $(n=10)$

Sit-down restaurants $(n=14)$

Other restaurant categories ( $n \leq 5$ for each)

\section{Finding and Representative Quotation}

Many children ate rice and/or noodles every day or almost every day: "Every day we eat rice, but sometimes we eat noodles."

Non-Asian breakfast foods were cereal with milk, doughnuts, muffins, sandwiches, pizza rolls, or other non-Asian foods: "For breakfast, I eat cereal with milk."

Asian breakfast foods included rice soup or porridge, cooked or made by a family member: "It could be cereal, or what my grandma makes which is ... a Chinese Tamale, or noodles, or porridge, or ... a seasoned rice mixture with like eggs, then chicken and scallops."

School foods included chicken nuggets, pizza, hash browns, fries, hamburgers, nachos, or spaghetti, eaten at least occasionally: "And for lunch, if I'm at school, I buy lunch at school. So I'm basically eating like french fries, and hamburgers and all that."

Three children did not like the foods served: "I usually get the salad because I don't really think the other stuff is good." "... It's not Asian."

Lunches brought from home were mostly sandwiches or Asian food: "Lunch, if it's school, would usually have a sandwich ... I don't buy it from school."

Reasons for bringing lunch included time/convenience $(n=4)$ and health $(n=3)$ : "The lunch lines at school are very long, so I don't want to waste my time standing in line." "The food that they [the school] prepare is kind of dirty. Once I ate it, and I had a stomach ache."

Children mentioned soup, noodles, or "meat and vegetables" (either together or separate): "Chinese food, like where it's served family style. You have your bowl of rice each person, and then you'd have some veggies and then some meats and then you'd just take your own."

Non-Asian dinner foods included quesadillas or grilled chicken with side salad and ranch dressing: "A typical dinner would be like ... grilled chicken, with a side salad ... sometimes I add ranch."

Drinks included water, fruit juices, and milk: "Water, I drink it every day."

Snacks included sweet or salty non-Asian snacks, or fruit: "I like these purple Doritos, I like Takis and hot Cheetos; I also like marshmallows, beef jerky, lollipops, cookies, and anything that's not chocolate ... not including M\&Ms [and] the Three Musketeers."

Examples of non-Asian fast-food restaurants and foods included McDonald's (hamburger, fries, and Coke), Chick-fil-A (chicken strips or soup), Domino's (pepperoni pizza), and KFC (fries and chicken wings): "My mom and I like Chick-fil-A soup ... so we sometimes go there."

Ambiguous Asian restaurants were restaurants for which children could not remember names or Asian restaurants in general: "My favorite restaurant probably would have to be Chinese restaurant ... The name is in Chinese. It's in Chinatown."

Non-Asian sit-down restaurants and foods included Red Lobster (lobster), Outback Steakhouse (steak, bread, and macaroni and cheese), and Olive Garden (pasta, bread, and soup): "Um, I like Red Lobster a lot ... I usually get two lobsters with the cheese dipping, the melted cheese, and with either broccoli or asparagus on the sides, and usually some shrimp."

Asian sit-down restaurants included local Chinese restaurants, with sample dishes being meatballs, lobster, fried chicken, steamed rice, or noodles: "Golden Palace ... I usually get fruit and fried chicken ... [and] lobster."

Other restaurant categories included non-Asian fast casual (eg, Chipotle), Asian buffet, Asian fast food/fast casual, non-Asian buffet (eg, CiCi's Pizza), and non-Asian ambiguous (eg, Italian): "I like going to Chipotle." "There's this other restaurant called Silver Palace. It's a buffet. I really like to go there 'cause they have really good mushrooms ... I get some crabs sometimes. I get some watermelons. There's ice cream there, too. And there's a specific egg soup that I really like." 
children ( $\mathrm{n}=8)$ and $50 \%$ of Americanborn children $(\mathrm{n}=8)$ brought lunch to school, compared with few lower-SES $(14 \% ; \mathrm{n}=1)$ or Asian-born (22\%; $\mathrm{n}=2$ ) children. Finally, dinner trends were similar to those for breakfast. All Asian-identified and most bicultural $(77 \% ; \mathrm{n}=10)$ children usually ate Asian dinners, vs 33\% of American-identified children $(\mathrm{n}=2)$. Children who ate non-Asian foods for dinner were either adopted by non-Asian parents or in multiracial households.

\section{Restaurants}

Overall, the frequency of eating at restaurants was low, ranging from once a week to only on special occasions. One child said, "Usually we have leftovers, so we don't really go out to restaurants, only if it's like special occasion." Another added, "We would usually always spend time at home making dinner."

Several restaurant categories emerged when children discussed their favorite restaurants. The most commonly mentioned, by 10 children, was nonAsian fast food. A total of 54\% of boys $(\mathrm{n}=7)$ mentioned fast-food restaurants, as opposed to $25 \%$ of girls $(n=3)$. Frequencies for consuming non-Asian fast food ranged from once a week to twice every 6 months.

Ten children mentioned ambiguous Asian restaurants, which were defined as Asian restaurants for which children could not remember names or Asian restaurants in general. Based on acculturation levels, $78 \%$ of Asian-born children $(\mathrm{n}=7)$ and $62 \%$ of bicultural children $(\mathrm{n}=8)$ ate at ambiguous Asian restaurants, compared with $19 \%$ of American-born, 17\% of Asian-identified, and $17 \%$ of American-identified children ( $\mathrm{n}=3,1$, and 1 , respectively). Frequencies for visiting ambiguous Asian restaurants ranged from twice a week to 5 times/y.

Fourteen children mentioned sitdown restaurants (ie, with table service), whether Asian or non-Asian. A total of 44\% percent of American-born children $(\mathrm{n}=7)$ mentioned non-Asian sit-down restaurants and 38\% $(n=6)$ mentioned Asian sit-down restaurants, compared with 1 Asian-born child (11\%) who mentioned a non-Asian sit-down restaurant and another who mentioned an Asian sit-down restaurant. There also appeared to be differences by SES, with half of higher SES $(47 \% ; \mathrm{n}=8)$ and no lower-SES children listing at least 1 non-Asian sit-down restaurant as their favorite, and several higher-SES (35\%; $\mathrm{n}=6)$ and 1 lower-SES ( $\mathrm{n}=14 \%$ ) mentioning Asian sit-down restaurants. Overall, children did not eat at sit-down restaurants frequently; the frequency ranged from once every other week to once every 5 months.

Less frequently mentioned restaurant categories included non-Asian fast casual, Asian buffet, Asian fast food/ fast casual, non-Asian buffet, and nonAsian ambiguous. Each was mentioned by $\leq 5$ children. Table 3 includes more details about restaurant findings.

\section{Grocery Stores}

When asked about grocery stores, almost half of participants $(\mathrm{n}=12)$ said their parents shopped at both Asian and non-Asian grocery stores. As 1 child elaborated,

We shop every Saturday, because I'm already in Chinatown because of Chinese school ..., so we just shop here [at a Chinese grocery store]. And sometimes we go to $\mathrm{H}-\mathrm{E}-\mathrm{B}$ [a dominant non-Asian grocery store in Texas] because it's real close to our house, in case we forget something.

Six children said their parents shopped at only Asian grocery stores, whereas 7 identified only non-Asian grocery stores. There appeared to be differences based on SES status: 7 higherSES (41\%) and no lower-SES participants shopped only at non-Asian grocery stores. The usual frequency for grocery shopping appeared to be once a week. Three children mentioned growing food, including mustard greens, okra, or green apples, in a home garden.

Foods commonly purchased at the grocery store were fruit/vegetables and meat/seafood, each mentioned by over half of participants. Other foods included grains, dairy, and snacks. Five children distinguished between foods purchased at Asian vs non-Asian grocery stores. As 1 child described, "In Chinese stores, she [mom] buys the fruits and vegetables because they're better than the American stores. And in the American stores, she buys the meat and bread." Another child similarly said that her grandparents purchased vegetables at Chinese grocery stores, whereas her family bought organic fruits on sale at H-E-B.

\section{Influences on Diet}

Almost all children $(\mathrm{n}=21)$ mentioned parents or family members as influences on what they ate. One child explained that his parents were "the ones that cook the food at home mostly," and 1 similarly said her mom was "the one who goes and buys stuff." Other children alluded to their parents being good role models ("Since they eat healthy stuff, I try to be healthy also"), influencing availability/accessibility of foods ("He's [Dad's] really buying fruits and vegetables"), or restricting foods ("My parents don't let me [eat chips] because of my teeth").

Six children mentioned health influences. For example, 1 child explained, "My family actually doesn't eat beef because it's kind of like a religion thing, but I eat it because I need the nutrition." Other health reasons included not eating desserts or sweets to promote dental health or not eating less healthful food because of eczema. One child explained that she wanted to be healthier overall and thus limited her consumption of ramen and Diet Coke.

Friends also influenced what children ate. Six children mentioned their friends; 1 child explained, "At school, I see them eat something, I really want to try it, so ... I go to the place where they got it, and I try it once and see if it's good or not." Four of the children who mentioned friends clarified that their friends influenced them occasionally, but not always. Most participants $(\mathrm{n}=14)$ believed they ate different things than their friends did: "We're different races so we eat different types of food" and "Their parents pack them ... not Chinese, they pack them sandwiches and sometimes nutrition bars." Despite these differences, 10 children acknowledged that they were not influenced by their friends or did not care what their friends ate.

Other influences on diet were school and special occasions (eg, parties, festivals). Five children believed that no one influenced what they ate, but when asked to elaborate, they mentioned their parents or friends. 


\section{DISCUSSION}

This study investigated Chinese American children's behaviors, food preferences, and cultural influences on diet, using qualitative interviews framed by the MDA. Key themes included (1) identifying both Asian and non-Asian as favorite foods; (2) consuming non-Asian foods for breakfast and lunch, but Asian foods for dinner; (3) not eating frequently at restaurants; (4) shopping at both Asian and non-Asian grocery stores; and (5) identifying family as influences on diet. These findings verified previous studies ${ }^{12-14,16,36,37}$ and added information from children's perspectives, also appearing to support the constructs in the MDA.

On the whole, children described their diets and associated behaviors as both Asian and non-Asian, which was consistent with other research in this area. ${ }^{12,14,16,36}$ As reported in previous studies, ${ }^{12,14,16}$ foods consumed for breakfast and snacks were mostly nonAsian, lunches were commonly eaten at school and included non-Asian school foods, and dinner meals included Asian foods with rice and/or noodles as staples. Common Asian foods mentioned in this study and elsewhere ${ }^{12}$ included rice, noodles, Asian vegetables, and soup, whereas common non-Asian foods included sweetened beverages, salty snacks, cereals, and beef patties. Acculturation appeared to have a role in Chinese American children's diets, although longitudinal research with a greater number and more diverse Asian American children could test whether acculturation is causally related to obesity and/or unhealthy dietary behaviors, and if so, in which populations.

There was low frequency of eating at restaurants, which was similar to another study with Asian American adults that found more than half of participants reporting occasional (1-2 times/wk) or infrequent (1-2 times/mo) dining out. ${ }^{13}$ Asian parents previously admitted to limiting the frequency of their family eating at restaurants, mainly because of cost and health. ${ }^{13}$ Because eating out of home may be a risk factor for intake of more energy and fat and fewer micronutrients, ${ }^{38}$ low frequency of eating at restaurants is a positive finding that needs to be encouraged and maintained among this population. However, many children listed fast-food restaurants as their favorites, so interventions are needed to educate children and their families on healthy choices at fast-food restaurants.

There were mixed findings regarding school lunch. Although most children ate school lunch, there appeared to be differences by age (ie, few middle school-aged students ate school lunch), and some children reported not liking school lunch (eg, because of taste preferences, perceived cleanliness). A few children in the current study, as well as Hmong American youth and adults in a previous study, also believed that school foods were unhealthy. ${ }^{36}$ Research is needed to understand why school lunch participation may decline in middle school, and to address children's perceptions regarding the cleanliness and appeal of school food.

Another finding was the importance of family members in influencing children's dietary behaviors, which was a theme among other qualitative studies with Asian American parents. ${ }^{12-14,36,37}$ In the current study, some children discussed parental modeling, food restrictions for health, environment, and other food parenting practices. ${ }^{39}$ Other studies reported parental expectations and encouragement. ${ }^{12,13,37}$ Given the importance of these practices on children's nutritional and weight status, ${ }^{40-42}$ and the scarcity of this research among Asian American families, ${ }^{43,44}$ more research is needed to investigate food parenting practices and the design of appropriate parenting interventions for this important group. Such research should test the cultural appropriateness of existing food parenting measures for Asian American parents, ${ }^{45}$ as well as investigate the relationship between food parenting practices and children's dietary behaviors and/or obesity. In a related manner, because middle schoolaged youth may be exposed to less parental control at home, have more autonomy, and be subject to more peer influence, an original assumption was that friends would be a major influence on children's diets in this study. However, many children in this study reported not being influenced by their friends or not caring what their friends ate, which was an unexpected finding. Although the interview questions, many of which focused on the home environment or family influences, may have resulted in greater emphasis on families than peers, the study's findings were consistent with other research showing that parents provided the strongest influence on children's behaviors, ${ }^{46}$ with peers being particularly influential in middle adolescence (ie, age 14-16 years). ${ }^{46,47}$ This study included participants in late childhood and early adolescence. These findings suggested that family, not friends, should be a major focus of interventions among children in this age range.

\section{Results suggest that family, not friends, should be a major focus of interventions among Chinese children in late childhood and early adolescence.}

Along with key themes, this study examined the intersection of themes with demographic and acculturation characteristics. Based on 2 proxy measures of acculturation (ie, birthplace and self-identified cultural rating), there appeared to be trends toward a more Westernized/non-Asian diet among more acculturated children (eg, eating non-Asian foods for breakfast, dining at non-Asian sit-down restaurants, not eating rice and/or noodles almost every day). Other research with Asian and Hispanic adults and children linked acculturation to less healthy dietary patterns (eg, greater soda intake) and obesity. ${ }^{48,49}$ These findings support the importance of taking acculturation and both Asian and non-Asian cultures into consideration when designing nutrition education programs for Chinese American children. In addition, higher-SES children had Westernized/non-Asian behaviors, including consuming non-Asian foods for breakfast, eating at nonAsian sit-down restaurants, or shopping at only non-Asian grocery stores. Similar to acculturation, lower SES may be independently related to lower diet quality (eg, more refined grains and added fats) and obesity. ${ }^{50,51}$ Given the interconnectedness of SES and acculturation, ${ }^{48}$ research is needed to differentiate the SES-diet relationship vs acculturation-diet relationship (and any interactions) to identify intervention targets better. Unfortunately, limitations resulting from the nature of qualitative data analysis, as well as NVivo's matrix coding query, 
precluded investigation of acculturation and SES interactions in the current study. There appeared to be overlap between high acculturation and high SES in the sample (Table 2), so there may have been potential overlap between acculturation and SES in themes.

Despite the contributions this study makes, there were several limitations. First, a small sample size precluded capturing all possible perspectives and experiences. However, data saturation was reached during analysis, which lent credibility to the findings. Similarly, some of the findings reflected the experiences of only a few children, not the majority. Nevertheless, these findings offer potentially important insights that may be used to generate hypotheses for future research in this area, and might have been endorsed by more children in a different or larger sample. Second, although the researchers made efforts to recruit participants with diverse backgrounds, acculturation levels, and ages, the convenience sample included mostly younger, Americanborn, bicultural-identified, or higherSES children. The relatively younger age of the sample (ie, most were aged 9 or 10 years) may have resulted in a greater emphasis on families and home environments, as well as more similarities to research with primary caregivers, whereas research with older adolescents, who have more autonomy, may have resulted in different findings. Next, as a cross-sectional study, the process of acculturation or dietary change could not be investigated as would be possible in a longitudinal study. Furthermore, acculturation was based on birthplace and self-identified cultural rating, which may not fully capture acculturation. The original intention was to use the SL-ASIA; unfortunately, there was little variability in the SLASIA scores in the current sample (ie, 18 of 25 children had a score of 3 ), so proxy measures, which had more variability, were used to assess acculturation. Research is needed to include participants with more diverse acculturation levels, as well as to assess whether the SL-ASIA can capture the variability in acculturation levels of Chinese American children. Finally, some of the interview questions may have inadvertently measured parental dietary preferences and practices; for example, it is unclear whether participants had a choice in whether they bought or brought school lunch, and if theybrought lunch, whether they chose the foods. Especially with younger children, parents have major roles in influencing children's behaviors and preferences, ${ }^{40-42}$ so it is difficult to separate children's practices and preferences from parental practices and preferences. Findings regarding children's dietary behaviors should be interpreted in the context of parental behaviors.

\section{IMPLICATIONS FOR RESEARCH AND PRACTICE}

The current study contributes to the limited research base on Chinese American children's behaviors, food preferences, and cultural influences on diet, using qualitative interviews framed by the MDA. Findings from this study can lead to the generation of hypotheses that could potentially inform the design of culturally appropriate nutrition education programs for the Chinese American population. Some of the themes and recommendations identified in this study (eg, choosing healthy Westernized foods, limiting the frequency of eating at restaurants, targeting families) are not specific to Chinese American children and may be applied more broadly to other American children. For example, this study provides support for designing interventions that target parents, grandparents, and extended family to encourage modeling and other effective food parenting practices. Interventions may also be designed to educate children about the risks associated with excessive fastfood consumption (eg, weight gain, increased energy intake ${ }^{52}$ ) and which foods to select at fast-food restaurants (reported by many participants as their favorite restaurants). Such interventions would be universally relevant.

However, some themes in the current study illuminated the need to incorporate intervention components that are unique to Chinese American children and families. For example, interventions targeting Chinese American children should take acculturation into consideration and thus encourage children to maintain healthy aspects of their Asian diets, while also incorporating healthy aspects of non-Asian diets and avoiding some of the less healthy aspects of Westernized diets.

Results suggest that
interventions for Chinese
American children should
address acculturation and
encourage healthy
aspects of Asian and
non-Asian diets.

Limited studies tested nutrition education programs in Chinese American children, ${ }^{53,54}$ so efforts are needed to identify effective and ineffective strategies for nutrition education in this population.

Findings from this study also have implications for nutrition education interventions regarding school foods offered to schools with large populations of Chinese American children. Within schools, appropriate interventions and messages are needed to address misperceptions about school meals that may hinder children from eating school meals (eg, perceived lack of cleanliness) and increase the appeal or cultural variety of foods served at school. The lack of Asian foods at school may be a barrier for

\section{Appropriate interventions and messages are needed to address misperceptions about school meals, including perceived lack of cleanliness.}

Asian children and parents that needs to be investigated, although another study of Chinese American parents found that all children ate Western foods, including those who brought lunch from home. ${ }^{14}$ A future study could test whether increasing the availability of Asian foods at school will increase the number of Asian American children who eat school meals. It is crucial to include parents in this research to understand their perceptions and behaviors, because children may not have a choice in whether they buy school lunch or bring lunch from home. 


\section{ACKNOWLEDGMENTS}

At the time of this study, CSD was supported fully by a Primary Care Research Training Grant from National Research Service Award (No. T32 HP10031). The authors thank all of the children and parents who participated in the study, as well as the staff members at the participating community/cultural centers. Thank you also to Tzu-An Chen for her help with recruitment, and Alicia Beltran for her help with NVivo.

\section{REFERENCES}

1. Murphy SL, Xu J, Kochanek KD. Deaths: Final Data for 2010. Hyattsville, MD: National Center for Health Statistics; 2013.

2. Swinburn BA, Caterson I, Seidell JC, James WP. Diet, nutrition and the prevention of excess weight gain and obesity. Public Health Nutr. 2004;7:123-146.

3. Donaldson MS. Nutrition and cancer: a review of the evidence for an anticancer diet. Nutr J. 2004;3:19.

4. Singer MR, Moore LL, Garrahie EJ, Ellison RC. The tracking of nutrient intake in young children: the Framingham Children's Study. Am J Public Health. 1995;85:1673-1677.

5. World Health Organization. Diet, Nutrition and the Prevention of Chronic Diseases: Report of a Joint WHO/FAO Expert Consultation. Geneva, Switzerland: World Health Organization; 2003.

6. US Census Bureau. Asians fastestgrowing race or ethnic group in 2012, Census Bureau Reports. http://www.cen sus.gov/newsroom/press-releases/2013/cb 13-112.html. Accessed October 20, 2015.

7. Centers for Disease Control and Prevention. CDC Health Disparities and Inequalities Report-United States, 2013. Atlanta, GA: Centers for Disease Control and Prevention; 2013.

8. Bates LM, Acevedo-Garcia D, Alegria M, Krieger N. Immigration and generational trends in body mass index and obesity in the United States: results of the National Latino and Asian American Survey, 2002-2003. Am J Public Health. 2008;98:70-77.

9. Hsu WC, Araneta MRG, Kanaya AM, Chiang JL, Fujimoto W. BMI cut points to identify at-risk Asian Americans for type 2 diabetes screening. Diabetes Care. 2015;38:150-158.

10. WHO Expert Consultation. Appropriate body-mass index for Asian popu- lations and its implications for policy and intervention strategies. Lancet. 2004;363:157-163.

11. Patil BS, Jayaprakasha GK, Vikram A. Indigenous crops of Asia and Southeast Asia: exploring health-promoting properties. HortScience. 2012;47:821-827.

12. Diep CS, Foster MJ, McKyer ELJ, Goodson P, Guidry JJ, Liew J. What are Asian-American youth consuming? A systematic literature review. J Immigr Minor Health. 2015;17:591-604.

13. Cluskey M, Edlefsen M, Olson B, et al. At-home and away-from-home eating patterns influencing preadolescents' intake of calcium-rich food as perceived by Asian, Hispanic and non-Hispanic white parents. J Nutr Educ Behav. 2008;40:72-79.

14. Lv N, Brown JL. Chinese American family food systems: impact of Western influences. J Nutr Educ Behav. 2010;42: 106-114.

15. US Census Bureau. Selected Population Profile in the United States: 2013 American Community Survey 1-year estimates. http://factfinder.census.gov/. Accessed August 20, 2015.

16. Demory-Luce DK, Morales M, Nicklas T. Acculturation, weight status, and eating habits among ChineseAmerican preschool children and their primary caregivers: a pilot study. Nutr Res. 2005;25:213-224.

17. Satia-Abouta J, Patterson RE, Neuhouser ML, Elder J. Dietary acculturation: applications to nutrition research and dietetics. J Am Diet Assoc. 2002;102:1105-1118.

18. Lutsey PL, Roux AVD, Jacobs DRJ, et al. Associations of acculturation and socioeconomic status with subclinical cardiovascular disease in the MultiEthnic Study of Atherosclerosis. Am J Public Health. 2008;98:1963-1970.

19. Chen L, Juon H-S, Lee S. Acculturation and BMI among Chinese, Korean and Vietnamese adults. J Community Health. 2012;37:539-546.

20. Satia-Abouta J. Dietary acculturation: definition, process, assessment, and implications. Int J Hum Ecol. 2003;4:71-86.

21. Hesketh K, Waters E, Green J, Salmon L, Williams J. Healthy eating, activity and obesity prevention: a qualitative study of parent and child perceptions in Australia. Health Promot Int. 2005;20:19-26.

22. Krølner R, Rasmussen M, Brug J, Klepp KI, Wind M, Due P. Determinants of fruit and vegetable consumption among children and adolescents: a review of the literature. Part II: qualitative studies. Int J Behav Nutr Phys Act. 2011;8:112.
23. Neumark-Sztainer D, Story M, Perry C, Casey MA. Factors influencing food choices of adolescents: findings from focus-group discussions with adolescents. J Am Diet Assoc. 1999;99:929-937.

24. Kirk S. Methodological and ethical issues in conducting qualitative research with children and young people: a literature review. Int J Nurs Stud. 2007;44: 1250-1260.

25. Gill P, Stewart K, Treasure E, Chadwick B. Conducting qualitative interviews with school children in dental research. Br Dent J. 2008;204:371-374.

26. Stewart K, Gill P, Treasure E, Chadwick B. Understandings about food among 6-11 year olds in South Wales. Food, Culture \& Society. 2006;9:317-336.

27. Dzewaltowski DA, Estabrooks PA, Johnston JA. Healthy Youth Places promoting nutrition and physical activity. Health Educ Res. 2002;17:541-551.

28. Lytle LA, Seifert S, Greenstein J, McGovern P. How do children's eating patterns and food choices change over time? Results from a cohort study. Am J Health Promot. 2000;14:222-228.

29. Suinn RM, Ahuna C, Khoo G. The Suinn-Lew Asian Self-Identity Acculturation scale: concurrent and factorial validation. Educ Psychol Meas. 1992;52: 1041-1046.

30. Fereday J, Muir-Cochrane E. Demonstrating rigor using thematic analysis: a hybrid approach of inductive and deductive coding and theme development. Int J Qual Methods. 2006;5:80-92.

31. Hsieh H-F, Shannon SE. Three approaches to qualitative content analysis. Qual Health Res. 2005;15:1277-1288.

32. Braun V, Clarke V. Using thematic analysis in psychology. Qual Res Psychol. 2006;3:77-101.

33. Frederick CB, Snellman K, Putnam RD. Increasing socioeconomic disparities in adolescent obesity. Proc Natl Acad Sci U S A. 2014;111:1338-1342.

34. Tandon PS, Zhou C, Sallis JF, Cain KL, Frank LD, Saelens BE. Home environment relationships with children's physical activity, sedentary time, and screen time by socioeconomic status. Int J Behav Nutr Phys Act. 2012;9:1-9.

35. US Department of Agriculture Food and Nutrition Service. School meals: income eligibility guidelines. http://www.fns. usda.gov/school-meals/income-eligibilityguidelines. Accessed May 9, 2016.

36. Kim LP, Harrison GG, KagawaSinger M. Perceptions of diet and physical activity among California Hmong adults and youths. Prev Chronic Dis. 2007;4:A93. 
37. Vue H, Reicks M. Individual and environmental influences on intake of calcium-rich food and beverages by young Hmong adolescent girls. J Nutr Educ Behav. 2007;39:264-272.

38. Lachat C, Nago E, Verstraeten R, Roberfroid D, Van Camp J, Kolsteren P. Eating out of home and its association with dietary intake: a systematic review of the evidence. Obes Rev. 2012;13:329-346.

39. Hughes SO, O'Connor TM, Power TG. Parenting and children's eating patterns: examining control in a broader context. Int J Child Adolesc health. 2008;1:323-329.

40. Gerards SMPL, Kremers SPJ. The role of food parenting skills and the home food environment in children's weight gain and obesity. Curr Obes Rep. 2015;4:30-36.

41. Clark HR, Goyder E, Bissell P, Blank L, Peters J. How do parents' child-feeding behaviours influence child weight? Implications for childhood obesity policy. J Public Health. 2007;29:132-141.

42. Birch LL, Davison KK. Family environmental factors influencing the developing behavioral controls of food intake and childhood overweight. Pediatr Clin North Am. 2001;48:893-907.
43. Pai H-L, Contento I. Parental perceptions, feeding practices, feeding styles, and level of acculturation of Chinese Americans in relation to their school-age child's weight status. Appetite. 2014;80:174-182.

44. Wehrly SE, Bonilla C, Perez M, Liew J. Controlling parental feeding practices and child body composition in ethnically and economically diverse preschool children. Appetite. 2014;73:163-171.

45. Hughes SO, Frankel LA, Beltran A, et al. Food parenting measurement issues: working group consensus report. Child Obes. 2013;9:S95-S102.

46. Patrick H, Nicklas TA. A review of family and social determinants of children's eating patterns and diet quality. J Am Coll Nutr. 2005;24:83-92.

47. Story M, Neumark-Sztainer D, French S. Individual and environmental influences on adolescent eating behaviors. J Am Diet Assoc. 2002;102:S40-S51.

48. Fitzgerald N. Acculturation, economic status, and health among Hispanics. NAPA Bull. 2010;34:28-46.

49. Diep CS, Lemaitre RN, Chen TA, et al. Acculturation and plasma fatty acid concentrations in Hispanic and
Chinese-American adults: the MultiEthnic Study of Atherosclerosis. PLoS One. 2016;11:e0149267.

50. Darmon N, Drewnowski A. Does social class predict diet quality? Am J Clin Nutr. 2008;87:1107-1117.

51. McLaren L. Socioeconomic status and obesity. Epidemiol Rev. 2007;29: 29-48.

52. St-Onge M-P, Keller KL, Heymsfield SB. Changes in childhood food consumption patterns: a cause for concern in light of increasing body weights. Am J Clin Nutr. 2003; 78:1068-1073.

53. Chen J-L, Weiss S, Heyman MB, Lustig RH. Efficacy of a child-centred and family-based program in promoting healthy weight and healthy behaviors in Chinese American children: a randomized controlled study. J Public Health (Oxf). 2010;32:219-229.

54. Chen JL, Kwan M, Mac A, Chin NC, Liu K. iStart smart: A primary-care based and community partnered childhood obesity management program for Chinese-American children: feasibility study. J Immigr Minor Health. 2013;15:1125-1128.

\section{Monthly Virtual Collections at JNEB}

Each month Shirley Camp, our New Resources editor, selects JNEB articles for a themed collection on a topic of interest to our readers. Recent topics have included survey validation, SNAP-Ed, nutrition and technology, heart health, farmers markets, and bone health. While the articles are from JNEB and are always available from the archive, each virtual collection is active for 2 months-check http://www.jneb.org often to see our newest virtual collections. 


\section{CONFLICT OF INTEREST}

The authors have not stated any conflicts of interest. 